\title{
Energy-Efficient Power Allocation for Underlay Cognitive Radio Systems
}

\author{
Lokman Sboui, Zouheir Rezki, and Mohamed-Slim Alouini \\ King Abdullah University of Science and Technology (KAUST), \\ Computer, Electrical and Mathematical Science and Engineering Division (CEMSE), \\ Thuwal 23955-6900, Saudi Arabia. \\ \{lokman.sboui, zouheir.rezki, slim.alouini\}@kaust.edu.sa
}

\begin{abstract}
We present a power allocation framework for spectrum sharing Cognitive Radio (CR) systems based on maximizing the energy efficiency (EE). First, we show that the relation between the EE and the spectral efficiency (SE) is strictly increasing in contrast with the SE-EE trade-off discussed in the literature. We also solve a non-convex problem and explicitly derive the optimal power for the proposed average EE under either a peak or an average power constraint. We apply our results to the underlay $C R$ systems where the power is limited by an additional interference constraint. When the instantaneous channel is not available, we provide a necessary and sufficient condition for the optimal power and present a simple sub-optimal power. In the numerical results, we show that the proposed EE corresponds to a higher $\mathrm{SE}$ at mid-range and high power regime compared to the classical EE. We also show that the sup-optimal solution is very close to the optimal solution. In addition, we deduce that the absence of instantaneous CSI affects the EE and the SE at high power regime compared to full CSI. In the CR context, we show that the interference threshold has a minimal effect on the EE compared to the SE.
\end{abstract}

Index Terms-Energy efficiency, spectral efficiency, optimal power allocation, underlay cognitive radio.

\section{INTRODUCTION}

Due to the continuous demand for high data rate wireless communication systems, many challenges appeared due to the shortage of the resources to satisfy the future need. From one side, the spectrum becomes scarce [2], and the cognitive radio (CR) paradigm was introduced to overcome this spectrum shortage [3], [4]. From another side, energy consumption becomes a global crucial concern in particular in the ICT and communication fields. In fact, the number of mobile subscribers is in an exponential increase and mobile operators need to optimize their expenses mainly governed by electricity bills. In addition, the carbon footprint of the ICT industry, in general, and the wireless industry, in particular, is in a continuous increase [5]. It is estimated that the ICT produces $2 \%$ of the global $\mathrm{CO}_{2}$ emission, which is equivalent to the aviation industry emission [6], [7], whereas cellular networks produces about $0.2 \%$ [8], [9]. These numbers raise a considerable concern especially with the expected growth in the coming years. Furthermore, energy efficiency is a

*Part of this work has been presented at the 2014 Personal, Indoor and Mobile Radio Communications (PIMRC'14), Washington DC, USA [1].

*This work was supported by the Qatar National Research Fund (a member of Qatar Foundation) under NPRP Grant NPRP 5-250-2-087. The statements made herein are solely the responsibility of the authors crucial issue in power-constrained applications such as mobile phones or wireless sensors in order to increase the life-cycle of these devices [10], [11]. CR underlay systems are also power-constrained by the level of interference allowed by the primary user [12]. In this setting, the power is simultaneously constrained by both interference and power constraints [13]. Consequently, researchers and industrials aim to overcome these challenges by developing energy efficient wireless communications labeled as "Green Communications" [8], [14].

In the literature, many works have focused on maximizing the spectral efficiency (SE) defined as the capacity or the achievable rate per unit bandwidth expressed in bits/s/Hz, e.g., [15], [16]. The power that maximizes the SE is not necessarily energy-efficient [17]. Hence a new metric, reflecting the EE, has to be defined. From an information theoretical perspective, the EE was first studied in [18] through the so-called minimum energy per information bit (J/bit). Recently, a new metric called energy efficiency (EE) was defined as the ratio of the rate over the power [17], [19]. It has been shown that maximizing the EE, given a particular power limit, does not lead to the maximum SE which introduces the SE-EE tradeoff [17], [20]-[22]. In fact, if the transmission power is solely considered, the maximum $\mathrm{EE}$ is realized for very low, almost zero power, [18], [23], [24]. These studies did not consider the circuit power which is independent of the transmission [19], [25], [26]. Adopting the circuit power changes the behavior of the EE from a strictly decreasing function of the SE to a bellshaped function where the maximum is given for a non-zero power [19]. In other studies, there was a focus on the amplifier power which was considered separately [27], [28] and was shown to be a function of the transmission power. In [27], it was shown that the total power consumption is proportional to the to the square root of the transmission power. In [28], a more general form is presented where the square root is generalized by an exponent between 0.5 and 0.64 . However, for simplicity, we assume that the total consumed power is the sum of a circuit power and the transmit power. In general, the circuit power is modeled as constant [25]. In some studies, the circuit power is modeled as a linear function of the data rate [29]. Note that the resulting EE is neither concave nor convex. In [26], the EE power allocation was presented after solving the non-convex optimization problem using fractional programming resulting in no explicit power expressions. Also, the authors in [26] assumed a perfect knowledge of the channel state information (CSI) at the transmitters and the 
receivers. Moreover, the authors in [30] also used the fractional programming to find the optimal power that maximizes the EE.

In this paper, we develop a new framework that considers the EE criterion over fading channels. We present explicit expressions of the optimal power under either a peak or an average power constraint. Obtaining these expressions is achieved via solving a non-convex optimization problem using the Lagrangian method instead of fractional programming, [26], [30]. We proceed by partitioning the solution space into convex sub-spaces. We also extend our study to the CR spectrum sharing scenario where there is an additional interference constraint on the power. We then study the case where the instantaneous CSI at the transmitter (CSI-T) is not available and only the channel statistics are available, i.e., statistical CSI. We present a sub-optimal, but less complex, power allocation that only depends on channel statistics.

Our main contributions, in this paper, are:

- Derivation of the optimal power that maximizes the instantaneous EE. The corresponding explicit EE and SE expressions are provided, and the SE-EE trade-off is discussed.

- Analysis of the proposed average EE power allocation with a peak or an average power constraint.

- Study the CR average EE power allocation with additional peak or an average interference constraint.

- Study the impact of CSI-T knowledge on the EE by computing the optimal power with statistical CSI, thus evaluating the performance gain provided by full CSI.

The rest of this paper is organized as follows. In Section II, the system model is presented. In Section III, the single user EE power is analyzed. The CR EE is studied in Section IV. In Section V, the EE power with statistical CSI-T is computed. Numerical results are presented in Section VI. Finally, the paper is concluded in Section VII.

\section{SySTEM MODEL}

In this paper, we consider two scenarios; (i) a preliminary scenario in which a single user communication, as depicted in Fig.1.a, is analyzed. (ii) an underlay spectrum sharing CR scenario in which a secondary user (SU) shares the spectrum with a primary user (PU) (Fig.1.b). We first develop an energy efficient power allocation framework with full CSI at both the transmitter and the receiver (CSI-TR), then study the case when the CSI at the transmitter (CSI-T) is not available.

In the single user communication scenario, the link is over a fading channel characterized by a complex gain $h$. The received signal is given by $r=h s+w$, where $r$ and $s$ are the received and transmitted signals, respectively, and $w$ is a zero-mean and unit-variance circularly symmetric complex white Gaussian noise which is independent of $h$. Let $\gamma=|h|^{2}$ the squared modulus of the channel gain and $\Omega$ the mean of $\gamma$, i.e., $\Omega=\mathbb{E}_{\gamma}[\gamma]$ where $\mathbb{E}_{\gamma}[\cdot]$ is the expectation operator with respect to the random variable $\gamma$. The channel can follow any distribution e.g., Rayleigh, Nakagami, Rician, etc. For illustration, we adopted the Rayleigh fading in the numerical results. We denote by $P$ the transmission power. This power can be limited either by a peak constraint, i.e., $P(\gamma) \leq P_{\text {peak }}$

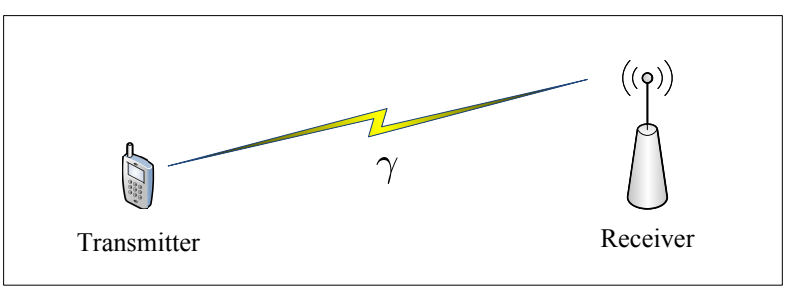

(a)

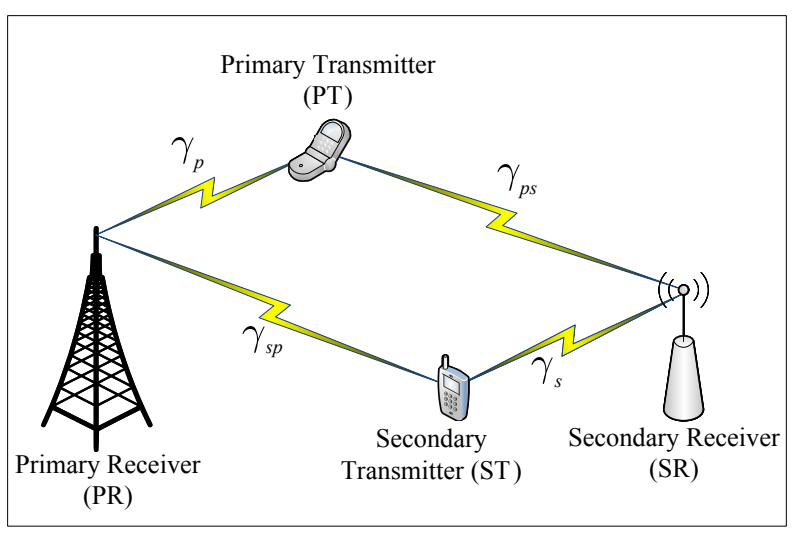

(b)

Figure 1: (a) Single User System Model (b) Underlay Cognitive Radio System Model.

or by an average constraint i.e., $\mathbb{E}_{\gamma}[P(\gamma)] \leq P_{\text {avg }}$, where $P_{\text {peak }}$ and $P_{\text {avg }}$ are the peak and the average power budgets, respectively. Our objective is to find the optimal power that maximizes the EE for a given channel realization $\gamma$. Before defining the EE, we recall the spectral efficiency SE which is an important performance measure for wireless communications. This measure is obtained by maximizing the Shannon capacity per unit bandwidth [16]. The EE is defined as the ratio of the SE over the consumed power which includes the transmit power $P$ and a circuit power $P_{c}$ [17], [19]. As we argue below, the circuit power $P_{c}$ is independent of the transmission and includes the consumption of cooling and all components [29]; analog to digital converters (ADC), filters, mixers, amplifiers, etc. The circuit power $P_{c}$ has a significant role in defining the optimal power that maximizes the EE. In fact, if $P_{c}=0$, the power that maximizes the EE tends toward zero [18] and the problem is directly solved. In the rest of the paper, we assume that $P_{c}>0$, hence the optimal power is different from zero. We first start by computing the optimal power for instantaneous $\mathrm{EE}$ then for average $\mathrm{EE}$.

Moreover, we extend our analysis to a CR scenario where the secondary transmitter (ST) is communicating with the secondary receiver (SR) under a tolerated interference threshold defined by the PU. Therefore, an interference constraint is set on the transmit power, and we analyze either a peak or an average interference constraint.

In our model, when the primary transmitter (PT) communicates with the primary receiver (PR), the SR is affected by the interference. We denote by $P_{p}$ the peak power of the PU transmission and by $h_{s}, h_{s p}, h_{p}$ and $h_{p s}$ the channel gains of 
the links ST-SR, ST-PR, PT-PR and PT-SR, respectively. We denote by $\gamma_{x}$ be the squared modulus of the channel $h_{x}$, i.e., $\gamma_{x}=\left|h_{x}\right|^{2}$ with $x \in\{s, s p, p, p s\}$. The objective is to provide an explicit expression of the optimal cognitive power that maximizes the $E E$ with respect to both power and interference constraints.

\section{Single User Energy Efficiency with Perfect CSI}

\section{A. Instantaneous Energy Efficiency}

The objective of maximizing the instantaneous EE is to find the corresponding optimal power and then generalize this result to the long term (average) EE. For a channel realization $\gamma$ we define the instantaneous spectral efficiency as

$$
S E=\log _{2}(1+\gamma P(\gamma)) \quad(\text { bps } / H z) .
$$

and the instantaneous energy efficiency (EE) as

$$
E E=\frac{\log _{2}(1+\gamma P(\gamma))}{P_{c}+P(\gamma)}(\text { bits } / J / H z) .
$$

Note that $E E$ is a positive function of $P(\gamma)$ and since $\lim _{P(\gamma) \rightarrow 0} E E=\lim _{P(\gamma) \rightarrow \infty} E E=0$ and the function is not multimodal, the function $E E$ has a global and unique maxima. Our objective is to find an explicit expression of the optimal power that maximizes the EE. The optimal power that maximizes the EE as defined by (2) is denoted $P_{E E}$ and is given by

$$
P^{*}=P_{E E}(\gamma)=\frac{1}{\gamma}\left(e^{1+W\left(\frac{\gamma P_{c}-1}{e}\right)}-1\right),
$$

where $W(\cdot)$ is the main branch of Lambert $\mathrm{W}$ function defined on $\left[-\frac{1}{e}, \infty\right)[31]$. Furthermore, the corresponding EE and SE are given by

$$
E E^{*}=\frac{\gamma}{\log (2) e^{1+W\left(\frac{\gamma P_{c}-1}{e}\right)}} \text { and } S E^{*}=\frac{1+W\left(\frac{\gamma P_{c}-1}{e}\right)}{\log (2)} .
$$

Proof: The proof is presented in Appendix A.

As a consequence of (3), and by adopting the optimal power $P_{E E}$ in (3) $\forall \gamma$, i.e., no power limit is set, we show in Appendix B, that

$$
E E^{*}=\frac{1}{P_{c}}\left(S E^{*}-\frac{1-2^{-S E^{*}}}{\log (2)}\right) .
$$

Note that the power expression in (3) is based on the Lambert function which has a lower complexity than the fractional programming method used generally to solve the problem. In addition, having explicit an expression of the power allows to extend the analysis and present the corresponding energy and spectral efficiencies as in (4). From (5), it can be verified easily that the $E E$ is an increasing function of $S E$. The Fig.2 depicts the instantaneous $\mathrm{EE}$ as a function of $\mathrm{SE}$ with different values of $\gamma$. The blue curves are the variation of $\mathrm{EE}$ and SE when the power spans all positive values for various values of $\gamma$. The red circles represent the optimal power solution, $P_{E E}$ that maximizes the EE. As can be seen in Fig.2, (5) represents a new EE-SE relation, presented by the dashed line, in which EE is a strictly increasing function of SE. Recall that

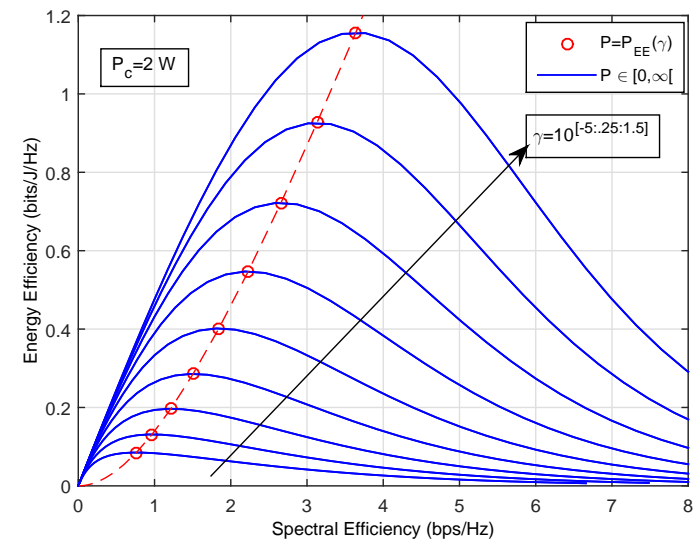

Figure 2: Instantaneous EE vs SE.

the trade-off between the EE and the SE [17], states that as the EE increases, the SE decreases. This result is true but only when the adopted power policy is the one that maximizes the SE. That is, this trade-off is observed when the SE criterion is considered in the power allocation scheme. However, when the power is driven by the $\mathrm{EE}$ criterion, increasing the $\mathrm{EE}$ leads to increasing the SE too, which means that there is no SE-EE trade-off in this case. We also analyze the asymptotic behavior of the EE with respect to the SE. From (5), when $S E \rightarrow 0$ we have $E E \approx \frac{\log (2) S E^{2}}{2 P_{c}}$ and when $S E \rightarrow \infty$ we have $E E \approx \frac{S E}{P_{c}}$. We note that the EE is proportional to the square of the SE at low SE values and becomes linear at high values. Also, the circuit power is an important parameter that defines the asymptotes of the EE at low and high SE values.

\section{B. Average Energy Efficiency}

The average EE considers the average performance over all channel realizations. In the literature, e.g., [26], the EE is defined as the ratio of the average rate and the average power. This classical expression of the EE describes the system energy efficiency but does not allow to tune the EE of the individual links [32]. As an alternative, another expression of the average $\mathrm{EE}$ is expressed as follows

$$
E E_{\text {avg }}=\mathbb{E}_{\gamma}\left[\frac{\log _{2}(1+\gamma P(\gamma))}{P_{c}+P(\gamma)}\right] .
$$

Note that the expression of the average EE in (6) is based on averaging the instantaneous EE of block fading channels. In addition, this expression takes into consideration the EE of each channel realization then averages these EE's instead of considering the system as a whole. This model of the EE is also called sum-EE defined in [32] as the arithmetic mean of the individual EE's [32]. Finally, we will show later in this paper that this expression of EE corresponds to a higher $\mathrm{SE}$ than the classical average $\mathrm{EE}$ at high power regimes. Meanwhile, once the power that maximizes $E E_{a v g}$ is found the corresponding spectral efficiency is given by:

$$
S E_{\text {avg }}=\mathbb{E}_{\gamma}\left[\log _{2}(1+\gamma P(\gamma))\right] .
$$


When no power constraint is set, it is clear that the optimal power that maximizes the average EE is the same as the one that maximizes the instantaneous EE, i.e., $P(\gamma)=P_{E E}(\gamma)$. However, when the power is either limited by a peak power constraint or an average power constraint, $P_{E E}(\gamma)$ may not always be the solution as we show in the next subsections.

1) Peak Power Constraint: In the peak power constraint, the transmit power is limited by a peak power noted $P_{\text {peak }}$ as follows

$$
P(\gamma) \leq P_{\text {peak }}, \forall \gamma>0
$$

In this case, in order to find the EE, we have to solve the following optimization problem

$$
\begin{array}{ll}
\max _{P(\gamma) \geq 0} & E E_{\text {avg }}=\mathbb{E}_{\gamma}\left[\frac{\log _{2}(1+\gamma P(\gamma))}{P_{c}+P(\gamma)}\right] . \\
& \text { subject to } P(\gamma) \leq P_{\text {peak }} .
\end{array}
$$

Note that, the optimal solution of this problem without considering the constraint (10) is $P_{E E}(\gamma)$. Hence if the power is limited by $P_{\text {peak }}$ the solution of the complete problem (9)-(10) is given by:

$$
P^{*}(\gamma)=\min \left\{P_{E E}(\gamma), P_{\text {peak }}\right\}
$$

where $P_{E E}$ is given in (3). Consequently, $E E_{a v g}$ is given by

$$
E E_{a v g}=\mathbb{E}_{\gamma}\left[\frac{\log _{2}\left(1+\gamma P^{*}(\gamma)\right)}{P_{c}+P^{*}(\gamma)}\right] \text {. }
$$

This expression can be explicitly computed once the p.d.f. of the fading channel is provided. In addition, at high values of $P_{\text {peak }}$, i.e., the optimal power is given by $P_{E E}(\gamma), \forall \gamma$, we have:

$$
\begin{aligned}
\lim _{P_{\text {peak }} \rightarrow \infty} E E_{\text {avg }} & =\frac{1}{\log (2)} \mathbb{E}_{\gamma}\left[\gamma e^{-\left(1+W\left(\frac{\gamma P_{c}-1}{e}\right)\right)}\right] . \\
\lim _{P_{\text {peak }} \rightarrow \infty} S E_{\text {avg }} & =\frac{1}{\log (2)}\left(1+\mathbb{E}_{\gamma}\left[W\left(\frac{\gamma P_{c}-1}{e}\right)\right]\right) .
\end{aligned}
$$

Furthermore, we study the convergence of the average EE with peak constraint to the average EE with no constraint for $P_{\text {peak }} \rightarrow \infty$. First, from [33], we have

$$
W(x) \underset{x \rightarrow \frac{-1}{e}}{\approx}-1+\sqrt{2(e x+1)} .
$$

Since, $e^{x} \underset{x \rightarrow 0}{\approx} 1+x$, we have

$$
\lim _{\gamma \rightarrow 0} P_{E E}(\gamma)=\lim _{\gamma \rightarrow 0} \frac{1}{\gamma}\left(e^{\sqrt{2 \gamma P_{c}}}-1\right)=\lim _{\gamma \rightarrow 0} \frac{\sqrt{2 P_{c}}}{\sqrt{\gamma}}=+\infty .
$$

Since $P_{E E}(\gamma)$ is monotonically decreasing in $\gamma$, then for any high value of $P_{\text {peak }}$, denoted $P_{\text {peak }}^{\infty}$, there exists a certain $\gamma^{\infty}>0$ such that for any $\gamma \in\left(0, \gamma^{\infty}\right)$, we have: $P_{E E}(\gamma)>$ $P_{\text {peak }}^{\infty}$ and hence the optimal power is $P^{*}=P_{p e a k}^{\infty}$. Thus, the condition

$$
P^{*}(\gamma)=P_{E E}(\gamma), \forall \gamma
$$

is never satisfied when a peak constraint is set. Hence, the average EE converges asymptotically to $\mathbb{E}_{\gamma}\left[E E^{*}(\gamma)\right]$ without attaining it.
2) Average Power Constraint: When the average power constraint is adopted, the transmit power is limited as follows

$$
\mathbb{E}_{\gamma}[P(\gamma)] \leq P_{\text {avg }}
$$

The average EE is obtained by solving the following optimization problem

$$
\begin{array}{cl}
\max _{P(\gamma) \geq 0} & E E_{\text {avg }}=\mathbb{E}_{\gamma}\left[\frac{\log _{2}(1+\gamma P(\gamma))}{P_{c}+P(\gamma)}\right], \\
& \text { subject to } \mathbb{E}_{\gamma}[P(\gamma)] \leq P_{\text {avg }} .
\end{array}
$$

Similarly to the previous cases, if no power constraint is set, the optimal solution is $P_{E E}(\gamma), \forall \gamma>0$. However, due to the average power constraint, we need to distinguish two cases in which the average power, $P_{a v g}$, is either smaller or greater than the average of $P_{E E}(\gamma)$, i.e., $\mathbb{E}_{\gamma}\left[P_{E E}(\gamma)\right]$.

- If $P_{a v g} \geq \mathbb{E}_{\gamma}\left[P_{E E}(\gamma)\right]$, the power that maximizes the instantaneous EE, i.e., $P^{*}(\gamma)=P_{E E}(\gamma), \forall \gamma>0$, is a feasible solution of the problem hence it is the solution of this problem since the constraint is inactive in this case. Thus, the corresponding $E E_{a v g}$ does not depend on $P_{a v g}$ and is given by the RHS of (13). Note that in this case, the $E E_{a v g}$ matches the asymptote of the peak constrained power case.

- If $P_{a v g}<\mathbb{E}_{\gamma}\left[P_{E E}(\gamma)\right]$, the optimal power is either equal to or smaller than $P_{E E}(\gamma), \forall \gamma>0$. Note that for a given $\gamma>0$, the optimal power cannot exceed $P_{E E}(\gamma)$ since it will result, in addition to decreasing the EE, in increasing the average power, in contradiction with the fact that $P_{a v g}<\mathbb{E}_{\gamma}\left[P_{E E}(\gamma)\right]$. Thus, $P^{*}(\gamma) \leq P_{E E}(\gamma), \forall \gamma>0$. We show that in this region of the power, $E E$ is an increasing and concave function with respect to $P(\gamma)$, (see Appendix C). Hence we can use the Lagrange method to solve this problem [34]. We compute the derivative of the Lagrangian function with respect to $P(\gamma)$. By using the method of variations, we obtain the following necessary and sufficient optimality condition:

$$
\begin{aligned}
& \frac{\gamma}{\log (2)(\gamma P(\gamma)+1)\left(P(\gamma)+P_{c}\right)}- \\
& \frac{\log (\gamma P(\gamma)+1)}{\log (2)\left(P(\gamma)+P_{c}\right)^{2}}=\lambda,
\end{aligned}
$$

where $\lambda$ is the Lagrange multiplier associated to the average power constraint. Note that if there is a certain $P^{*}(\gamma)$ verifying $(20), \forall \gamma>0$, then $P^{*}(\gamma)$ is the optimal power that maximizes the EE. Note that since $\lambda \geq 0$ and $P_{E E}(\gamma)$ is the power that cancels out the LHS of (20), then the set of powers such as $P(\gamma)>P_{E E}(\gamma)$ will result in a negative LHS of (20). Hence, the condition $P(\gamma) \leq P_{E E}(\gamma)$ is always satisfied for $\lambda \geq 0$. In order to find $P^{*}(\gamma)$, we define the function

$$
\begin{aligned}
& G_{\gamma, P_{c}}(x)= \\
& \frac{\gamma}{\log (2)(\gamma x+1)\left(x+P_{c}\right)}-\frac{\log (\gamma x+1)}{\log (2)\left(x+P_{c}\right)^{2}} .
\end{aligned}
$$

In fact, $G_{\gamma, P_{c}}(P)=\frac{\partial E E}{\partial P}$ and $\frac{\partial G_{\gamma, P_{c}}}{\partial P}=\frac{\partial^{2} E E}{\partial P^{2}}$. Since $E E(P)$ is concave, for $P(\gamma)<P_{E E}(\gamma)$, then we have 
$\frac{\partial^{2} E E}{\partial P^{2}}<0$ thus $\frac{\partial G_{\gamma, P_{c}}}{\partial P}<0$. We have a continuous and a strictly decreasing function $G_{\gamma, P_{c}}(\cdot)$, then $G_{\gamma, P_{c}}(\cdot)$ is a bijection on $\left[0, P_{E E}(\gamma)\right]$ and, consequently, has an inverse denoted $G_{\gamma, P_{c}}^{-1}(\cdot)$. Thus, for a fixed $\lambda_{0}$ and $\gamma$, there is a unique positive $P_{P_{\text {avg }}}(\gamma)$ such that

$$
P_{P_{a v g}}(\gamma)=\left\{\begin{array}{l}
G_{\gamma, P_{c}}^{-1}\left(\lambda_{0}\right) \text { if } \lambda_{0} \in\left(0, \frac{\gamma}{\log (2) P_{c}}\right) \\
0 \quad \text { otherwise }
\end{array}\right.
$$

Thus, we have

$$
P_{\text {avg }}=\mathbb{E}_{\gamma}\left[P_{P_{a v g}}(\gamma)\right] .
$$

Finally, to find the optimal solution for a given $P_{a v g}$, we determine the corresponding Lagrangian multiplier using (23). Then, we compute the optimal power by solving (20) for each value of $\gamma$.

In summary, the optimal power that maximizes the average $\mathrm{EE}$, with an average power constraint, is given by

$$
P^{*}(\gamma)=\left\{\begin{array}{l}
P_{E E}(\gamma) \text { if } P_{a v g} \geq \mathbb{E}_{\gamma}\left[P_{E E}(\gamma)\right] \\
P_{P_{a v g}} \text { otherwise. }
\end{array}\right.
$$

Note that for high values of $P_{a v g}$, the power is equal to $P_{E E}(\gamma) \forall \gamma>0$. Hence, the EE and SE asymptotes are the same as those given by (13) and (14), respectively. However, unlike the peak power-constrained scenario, these asymptotes are reached once $P_{a v g}$ is higher than $\mathbb{E}_{\gamma}\left[P_{E E}(\gamma)\right]$.

3) Effect of Low Circuit Power: We study the effect of low circuit power, i.e., $P_{c} \rightarrow 0$, on the system performances EE and SE independently from the adopted constraints. When $P_{c} \rightarrow 0$, we have from (3), $P_{E E} \rightarrow 0$ which means that the $E E$ is maximal in the asymptotically low power regime. In this case, the corresponding $E E_{a v g}$ and $S E_{a v g}$ tend towards $\frac{\Omega}{\log (2)}$ and 0 , respectively [18]. Of course, this case is not practical but it shows that whenever $P_{c} \rightarrow 0$, maximizing the $E E$ will lead to a reduced throughput if the EE criterion is adopted for the power allocation. Note that $\frac{\Omega}{\log (2)}$ presents the maximum value $E E_{a v g}$ can reach [19] as a function of $P_{c}$ for a given channel mean $\Omega$. As $P_{c}$ increases, the maximum of $E E_{a v g}$ decreases as can be shown after averaging the instantaneous $E E^{*}$ in (4). In this Section, we presented the optimal power allocation for instantaneous and average $\mathrm{EE}$ in a point to point scenario. In the next Section, we generalize our results to $\mathrm{CR}$ systems.

\section{Cognitive Radio Energy EfFiciency with PERFECT CSI}

In this section, we aim to compute the optimal power allocation scheme that maximizes the EE in a spectrum sharing underlay CR context shown in Fig.1(b). The corresponding instantaneous $\mathrm{EE}$ at the $\mathrm{SU}$ is given by:

$$
E E=\frac{\log _{2}\left(1+\frac{\gamma_{s} P_{s}}{1+\gamma_{p s} P_{p}}\right)}{P_{c}+P_{s}} .
$$

where $P_{s}$ is the allocated power at the ST and $P_{p}$ is the peak power at the PT. By adopting $P_{p}$, we analyze the worst-case secondary performance independently from the primary power variation. Note that $P_{s}$ is, in general, a function of many parameters, such as $\gamma_{s}, \gamma_{s p}, \gamma_{p s}, P_{p}$, i.e., $P_{s}\left(\gamma_{s}, \gamma_{s p}, \gamma_{p s}, P_{p}\right)$. In this Section, we assume that the CSI related to the ST, i.e. $\gamma_{s}$ and $\gamma_{s p}$, are estimated perfectly whereas the CSI related to the PT, i. e. $\gamma_{p s}$, is provided to the ST via a feedback link. Arguably, this feedback mechanism may be considered as a mean to capture a cooperative primary network rewarded via some incentives by the secondary network. This assumption leads to an upper bound on the cognitive achievable rate. However, due to CSI limitations, some of these parameters cannot be available at the ST, which will be analyzed in the next Section. For simplicity, the secondary transmit power will be denoted by $P_{s}$ with no arguments. In the underlay CR scenario, an additional interference constraint is adopted to protect the PU communication from the eventual interference caused by the ST. This constraint can be either a peak or an average constraint. We study the optimal power allocation corresponding to each constraint as follows:

\section{A. Peak Interference and Power constraints}

The peak interference constraint protects the PR instantaneously and ensures that the ST interference on the PR is below the threshold for all channel realizations [35]. The expression of the peak interference constraint is given by:

$$
\gamma_{s p} P_{s} \leq I_{\text {peak }}
$$

where $I_{\text {peak }}$ is the tolerated interference threshold allowed by the PR. The corresponding optimization problem is given by

$$
\begin{aligned}
\max _{P_{s} \geq 0} E E_{\text {avg }}= & \mathbb{E}_{\gamma_{p s}, \gamma_{s}}\left[\frac{\log _{2}\left(1+\frac{\gamma_{s} P_{s}}{1+\gamma_{p s} P_{p}}\right)}{P_{c}+P_{s}}\right] \\
\text { subject to } & P_{s} \leq P_{\text {peak }} ; \\
& \gamma_{s p} P_{s} \leq I_{\text {peak }} .
\end{aligned}
$$

The optimization problem in (27)-(29) can be written as:

$$
\begin{aligned}
& \max _{P_{s} \geq 0} E E_{a v g}=\mathbb{E}_{\tilde{\gamma}_{s}}\left[\frac{\log _{2}\left(1+\tilde{\gamma}_{s} P_{s}\right)}{P_{c}+P_{s}}\right] \\
& \quad \text { subject to } P_{s} \leq \tilde{P}_{\text {peak }},
\end{aligned}
$$

where $\tilde{\gamma}_{s}=\frac{\gamma_{s}}{1+\gamma_{p s} P_{p}}$ and $\tilde{P}_{\text {peak }}=\min \left\{P_{\text {peak }}, \frac{I_{p e a k}}{\gamma_{s p}}\right\}$. Note that in this case, $P_{s}$ is a function of $\gamma_{s}, \gamma_{s p}, \gamma_{p s}, P_{p}$. Hence from (11), the solution of this problem is

$$
\begin{aligned}
P_{s}^{*} & =\min \left\{P_{E E}\left(\tilde{\gamma}_{s}\right), \tilde{P}_{\text {peak }}\right\} \\
& =\min \left\{\frac{1}{\tilde{\gamma}_{s}}\left(e^{1+W\left(\frac{\tilde{\gamma}_{s} P_{c}-1}{e}\right)}-1\right), P_{\text {peak }}, \frac{I_{\text {peak }}}{\gamma_{s p}}\right\} .
\end{aligned}
$$

Note that this solution means that in case the two constraints are inactive, i.e., $P_{E E}\left(\tilde{\gamma}_{s}\right) \leq \tilde{P}_{\text {peak }}$, the EE reaches its highest value since the allocated power is the one that maximizes the $\mathrm{EE}$ as shown in (3).

\section{B. Average Interference and Power Constraints}

The average interference constraint allows to protect the long term PU communication [36]. In fact, this type of interference constraint is less strict than the peak interference constraint since the SU power can exceed the interference 
threshold for some channel realizations. The average interference constraint is given by

$$
\mathbb{E}_{\gamma_{p s}, \gamma_{s}}\left[\gamma_{s p} P_{s}\right] \leq I_{a v g}
$$

The corresponding optimization problem is given by

$$
\begin{aligned}
\max _{P_{s} \geq 0} E E_{\text {avg }} & =\mathbb{E}_{\tilde{\gamma}_{s}}\left[\frac{\log _{2}\left(1+\tilde{\gamma}_{s} P_{s}\right)}{P_{c}+P_{s}}\right] \\
\text { subject to } & \mathbb{E}_{\tilde{\gamma}_{s}}\left[P_{s}\right] \leq P_{a v g} ; \\
& \mathbb{E}_{\tilde{\gamma}_{s}, \gamma_{s p}}\left[\gamma_{s p} P_{s}\right] \leq I_{a v g} .
\end{aligned}
$$

Note that similarly to Section (III-B2), $P_{E E}\left(\tilde{\gamma}_{s}\right)$ is optimal if no constraints are set. However, in presence of (36) and (37), there are 4 cases which have to be distinguished:

1) $\mathbb{E}_{\tilde{\gamma}_{s}}\left[P_{E E}\left(\tilde{\gamma}_{s}\right)\right] \leq P_{a v g}$ and $\mathbb{E}_{\tilde{\gamma}_{s}}\left[\gamma_{s p} P_{E E}\left(\tilde{\gamma}_{s}\right)\right] \leq I_{a v g}$ : In this region, the solution of the problem is $P^{*}=P_{E E}\left(\tilde{\gamma}_{s}\right)$ since the constraints are inactive.

2) $\mathbb{E}_{\tilde{\gamma}_{s}}\left[P_{E E}\left(\tilde{\gamma}_{s}\right)\right] \leq P_{a v g}$ and $\mathbb{E}_{\tilde{\gamma}_{s}}\left[\gamma_{s p} P_{E E}\left(\tilde{\gamma}_{s}\right)\right] \geq I_{a v g}$ : The constraint (37) forces the power to be below $P^{*}=P_{E E}\left(\tilde{\gamma}_{s}\right)$, however using the convexity of the EE in this region, we can use the Lagrangian method to solve the problem. After computing the Lagrange function, we obtain the following necessary and sufficient optimality condition:

$$
\frac{\tilde{\gamma}_{s}}{\log (2)\left(\tilde{\gamma}_{s} P_{s}+1\right)\left(P_{s}+P_{c}\right)}-\frac{\log \left(\tilde{\gamma}_{s} P_{s}+1\right)}{\log (2)\left(P_{s}+P_{c}\right)^{2}}=\mu \gamma_{s p},
$$

where $\mu$ is the Lagrange multiplier associated to the average interference constraint. Similarly to Section III-B2, by using the bijection of $G_{\tilde{\gamma}_{s}, P_{c}}^{-1}(\cdot)$ defined in (21), for a fixed $\mu_{0}$, there is a unique positive power denoted $P_{I_{a v g}}$ such as

$$
P_{I_{a v g}}=\left\{\begin{array}{l}
G_{\tilde{\gamma}_{s}, P_{c}}^{-1}\left(\mu_{0} \gamma_{s p}\right) \text { if } \mu_{0} \gamma_{s p} \in\left(0, \frac{\tilde{\gamma}_{s}}{\log (2) P_{c}}\right), \\
0 \quad \text { otherwise. }
\end{array}\right.
$$

The Lagrange multiplier $\mu$ is obtained by satisfying the active constraint with equality. That is,

$$
I_{a v g}=\mathbb{E}_{\tilde{\gamma}_{s}}\left[\gamma_{s p} P_{I_{a v g}}\right] .
$$

Finally, to find the optimal solution for a given $I_{a v g}$, we determine, numerically, the corresponding Lagrangian multiplier $\mu$ using (40). Then, we compute the optimal power by solving (39).

3) $\mathbb{E}_{\tilde{\gamma}_{s}}\left[P_{E E}\left(\tilde{\gamma}_{s}\right)\right] \geq P_{a v g}$ and $\mathbb{E}_{\tilde{\gamma}_{s}}\left[\gamma_{s p} P_{E E}\left(\tilde{\gamma}_{s}\right)\right] \leq I_{a v g}$ : In this region, the constraint (36) forces the power to be below $P^{*}=P_{E E}\left(\tilde{\gamma}_{s}\right)$. Hence, the solution has the same shape as in (24), by substituting $\gamma$ by $\tilde{\gamma}_{s}$ and $P_{P_{a v g}}$ is computed using the function $G_{\tilde{\gamma}_{s}, P_{c}}^{-1}(\cdot)$ instead of $G_{\gamma, P_{c}}^{-1}(\cdot)$.

4) $\mathbb{E}_{\tilde{\gamma}_{s}}\left[P_{E E}\left(\tilde{\gamma}_{s}\right)\right] \geq P_{a v g}$ and $\mathbb{E}_{\tilde{\gamma}_{s}}\left[\gamma_{s p} P_{E E}\left(\tilde{\gamma}_{s}\right)\right] \geq I_{a v g}$ : In this region, the power is affected by both the average power and the interference thresholds, i.e., $P_{a v g}$ and $I_{a v g}$, respectively. Hence, we use the Lagrangian method, and we obtain the following sufficient optimality condition

$\frac{\tilde{\gamma}_{s}}{\log (2)\left(\tilde{\gamma}_{s} P_{s}+1\right)\left(P_{s}+P_{c}\right)}-\frac{\log \left(\tilde{\gamma}_{s} P_{s}+1\right)}{\log (2)\left(P_{s}+P_{c}\right)^{2}}=\lambda+\mu \gamma_{s p}$,

where $\lambda$ and $\mu$ are the Lagrange multiplier associated to the average power and average interference constraints, respectively. Consequently, from the previous results and by using the function $G_{\tilde{\gamma}_{s}, P_{c}}(\cdot)$, the optimal power, for a fixed $\lambda_{0}$ and $\mu_{0}$ is

$$
P_{P_{a v g}, I_{a v g}}=\left(G_{\tilde{\gamma}_{s}, P_{c}}^{-1}\left(\lambda_{0}+\mu_{0} \gamma_{s p}\right)\right)^{+},
$$

where $(\cdot)^{+}$is $\max \{0,$.$\} . Using the slackness conditions, the$ multipliers $\mu_{0}$ and $\lambda_{0}$ are determined such as

$$
\begin{array}{r}
\mathbb{E}_{\tilde{\gamma}_{s}}\left[\gamma_{s p}\left(G_{\tilde{\gamma}_{s}, P_{c}}^{-1}\left(\mu_{0} \gamma_{s p}\right)\right)^{+}\right]=I_{a v g} \\
\text { and } \mathbb{E}_{\gamma}\left[\left(G_{\gamma, P_{c}}^{-1}\left(\lambda_{0}\right)\right)^{+}\right]=P_{a v g} .
\end{array}
$$

Hence, to find the optimal power in this region, we determine the Lagrange multipliers form (43), then compute the power using (41). Table I summarizes the solutions of the average constraints in the different regions.

\section{Peak Interference and Average Power Constraints}

If we adopt an average power and peak interference constraint, the optimal power becomes

$P_{s}^{*}= \begin{cases}\min \left\{P_{E E}\left(\tilde{\gamma}_{s}\right), \frac{I_{p e a k}}{\gamma_{s p}}\right\} & \text { if } P_{a v g} \geq \mathbb{E}_{\tilde{\gamma}_{s}}\left[P_{E E}\left(\tilde{\gamma}_{s}\right)\right], \\ \min \left\{P_{P_{a v g}}, \frac{I_{p e a k}}{\gamma_{s p}}\right\} & \text { otherwise. }\end{cases}$

In order to obtain (44), we use similar techniques that led to (24) with the introduction of the peak interference threshold $I_{\text {peak }}$.

\section{Average Interference and Peak Power Constraints}

Similarly to the previous cases, if we adopt a peak power and average interference constraints, the optimal power becomes

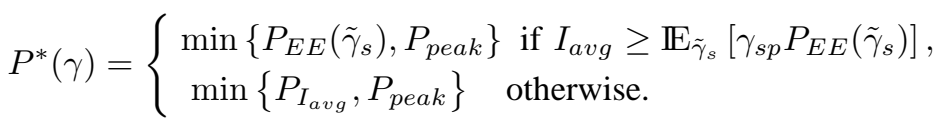

To establish (45), we use once again similar techniques as in the previous subsections. Details of the proof are skipped for brevity.

\section{Average Energy EfFiciency With Statistical CSI}

\section{A. Single User Energy Efficiency}

1) No Power Constraint: In the case where the CSI is not available, the transmitter is not able to adapt its power with respect to the instantaneous channel gain. Instead, the power will depend on the channel gain statistics. If no power constraint is set, the optimal power that maximizes the average $E E_{\text {avg }}=\mathbb{E}_{\gamma}\left[\frac{\log _{2}(1+\gamma P)}{P_{c}+P}\right]$ is found by solving the following equation:

$$
\mathbb{E}_{\gamma}\left[\frac{\gamma}{1+\gamma P}\right]-\frac{1}{P_{c}+P} \mathbb{E}_{\gamma}\left[\log _{2}(1+\gamma P)\right]=0 .
$$




\begin{tabular}{|c|c|c|}
\hline Regions & $\mathbb{E}_{\tilde{\gamma}_{s}}\left[P_{E E}\left(\tilde{\gamma}_{s}\right)\right] \geq P_{a v g}$ & $\mathbb{E}_{\tilde{\gamma}_{s}}\left[P_{E E}\left(\tilde{\gamma}_{s}\right)\right] \leq P_{a v g}$ \\
\hline $\mathbb{E}_{\tilde{\gamma}_{s}}\left[\gamma_{s p} P_{E E}\left(\tilde{\gamma}_{s}\right)\right] \geq I_{a v g}$ & $P_{P_{a v g}, I_{a v g}}$ & $P_{I_{a v g}}$ \\
\hline $\mathbb{E}_{\tilde{\gamma}_{s}}\left[\gamma_{s p} P_{E E}\left(\tilde{\gamma}_{s}\right)\right] \leq I_{a v g}$ & $P_{P_{a v g}}$ & $P_{E E}\left(\tilde{\gamma}_{s}\right)$ \\
\hline
\end{tabular}

Table I: CR optimal power with average interference and power constraints

Unfortunately, the equation (46), does not lead to a closedform, but can be solved numerically to find the optimal power. Instead of solving (46), one may be interested in

$$
E E_{\text {avg }}=\mathbb{E}_{\gamma}\left[\frac{\log _{2}(1+\gamma P)}{P_{c}+P}\right] \leq \frac{\log _{2}\left(1+\mathbb{E}_{\gamma}[\gamma] P\right)}{P_{c}+P} .
$$

Now, instead of maximizing the left hand side (LHS) of (47), it is more convenient to maximize the right hand side (RHS) of (47). Therefore, the problem becomes similar to the instantaneous EE problem in (2) and the corresponding solution can be explicitly computed from (3) as follows

$$
P^{*}=P_{E E}\left(\mathbb{E}_{\gamma}[\gamma]\right)=P_{E E}(\Omega) .
$$

This solution presents a simple power allocation that, when applied to the original function, i.e., $E E_{\text {avg }}$, gives a lower bound of the secondary EE since it is not necessary the optimal for the $E E_{\text {avg }}$. We show later, in the numerical results, that this solution gives a good lower bound, at least for Rayleigh fading. In the rest of the paper, this sub-optimal solution will be adopted in the statistical CSI cases where either a peak or an average power constraint is set.

2) Peak Power Constraint: In this scenario, the corresponding sub-optimal power is given by the minimum of the peak power, $P_{\text {peak }}$ and the sub-optimal power in (48) as follows

$$
P^{*}(\gamma)=\min \left\{P_{E E}(\Omega), P_{\text {peak }}\right\} .
$$

3) Average Power Constraint: Since the power does not depend on $\gamma$, the average constraint becomes similar to a peak constraint as follows, i.e., $P \leq P_{\text {avg }}$. Consequently, the corresponding optimal power under average power constraint, is given by

$$
P^{*}(\gamma)=\min \left\{P_{E E}(\Omega), P_{\text {avg }}\right\} .
$$

Note that if we target the optimal power when the CSI is not provided, we just substitute the sub-optimal solution $P_{E E}(\Omega)$ by the solution of (46), computed numerically, in (49) and (50) for peak and average power constraints, respectively. However, in the numerical results we show that the gap between the optimal and sub-optimal EE is very small for Rayleigh fading.

\section{B. Cognitive Energy Efficiency}

In the CR context, the absence of the CSI happens when there is neither instantaneous cooperation from the PR nor instantaneous feedback from the SR. In the case of no cooperation, the ST does not have the instantaneous values of $\gamma_{s p}$ but (by an implicit assumption) knows its average $\Omega_{s p}$. Consequently, the ST transmits with the power in (33), but with substituting $\gamma_{s p}$ by $\Omega_{s p}$. Meanwhile, when there is no feedback from the SR about $\gamma_{s}$ and $\gamma_{s p}$, the ST transmits with the power that involves their statistics, i.e., $\Omega_{s}$ and $\Omega_{s p}$. When the CSI is not available in the CR setting, only an average interference constraint can be applied since the peak constraint requires instantaneous value of the channel gain. In this section, we analyze two setting in which the power constraint is either peak or average.

1) Peak Power Constraint: When only statistical CSI is available, the corresponding problem involving peak power and average interference constraint can be written as follows

$$
\begin{aligned}
\max _{P_{s} \geq 0} E E_{\text {avg }} & =\mathbb{E}_{\tilde{\gamma}_{s}}\left[\frac{\log _{2}\left(1+\tilde{\gamma}_{s} P_{s}\right)}{P_{c}+P_{s}}\right] \\
\text { subject to } & P_{s} \leq P_{\text {peak }} ; \\
& \mathbb{E}_{\gamma_{s p}}\left[\gamma_{s p} P_{s}\right] \leq I_{\text {avg }} .
\end{aligned}
$$

We notice that the average interference constraint can be written as a peak constraint since $\mathbb{E}_{\gamma_{s p}}\left[\gamma_{s p} P_{s}\right]=\Omega_{s p} P_{s}$. Hence, (53) becomes $\Omega_{s p} P_{s} \leq I_{a v g}$. Consequently, adopting the power in (49), the solution of the CR problem is

$$
P_{s}^{*}=\min \left\{\frac{1}{\tilde{\Omega}_{s}}\left(e^{1+W\left(\frac{\tilde{\Omega}_{s} P_{c}-1}{e}\right)}-1\right), P_{\text {peak }}, \frac{I_{\text {peak }}}{\Omega_{s p}}\right\}
$$

where $\tilde{\Omega}_{s}=\mathbb{E}_{\tilde{\gamma}_{s}}\left[\tilde{\gamma}_{s}\right]=\mathbb{E}_{\gamma_{s}, \gamma_{s p}}\left[\frac{\gamma_{s}}{1+\gamma_{s p} P_{p}}\right]$

2) Average Power Constraint: When only statistical CSI is available, the average power constraint becomes a peak interference since the transmit power does not depend on the instantaneous channel gain and hence the problem becomes:

$$
\begin{aligned}
\max _{P_{s} \geq 0} E E_{\text {avg }} & =\mathbb{E}_{\tilde{\gamma}_{s}}\left[\frac{\log _{2}\left(1+\tilde{\gamma}_{s} P_{s}\right)}{P_{c}+P_{s}}\right] \\
\text { subject to } & \mathbb{E}_{\tilde{\gamma}_{s}}\left[P_{s}\right]=P_{s} \leq P_{\text {avg }} ; \\
& \mathbb{E}_{\gamma_{s p}}\left[\gamma_{s p} P_{s}\right]=\Omega_{s p} P_{s} \leq I_{\text {avg }}
\end{aligned}
$$

which is similar to the peak constraint problem and, hence, the solution is

$$
P_{s}^{*}=\min \left\{\frac{1}{\tilde{\Omega}_{s}}\left(e^{1+W\left(\frac{\tilde{\Omega}_{s} P_{c}-1}{e}\right)}-1\right), P_{a v g}, \frac{I_{a v g}}{\Omega_{s p}}\right\} .
$$

\section{Numerical Results}

We adopt a Rayleigh fading channel where the p.d.f. of $\gamma$ is given by the exponential distribution, i.e., $f_{\gamma}=e^{-\gamma}$. We denote by $X_{E E_{-} \text {criterion }} X_{S E_{-} \text {criterion }}$ the performance $X$ considering the $\mathrm{SE}$ and the EE criteria, respectively, were $X \in\{E E, S E\}$. In Fig. 3, the EE and the SE are presented as functions of the instantaneous channel realization $\gamma$. We first highlight the impact of limiting the power when adopting the EE criterion. We compare the unconstrained performances, i.e., $P(\gamma)=P_{E E}(\gamma)$, in continuous lines with peak powerconstrained performances, i.e., $P(\gamma)=\min \left\{P_{E E}(\gamma), P_{\text {peak }}\right\}$, in dot-dashed curves, with $P_{\text {peak }}=6 \mathrm{~dB}$. We notice that since $\lim _{\gamma \rightarrow 0} P_{E E}(\gamma)=+\infty$, limiting the power with peak power results in clipping the power for low values of $\gamma$. 


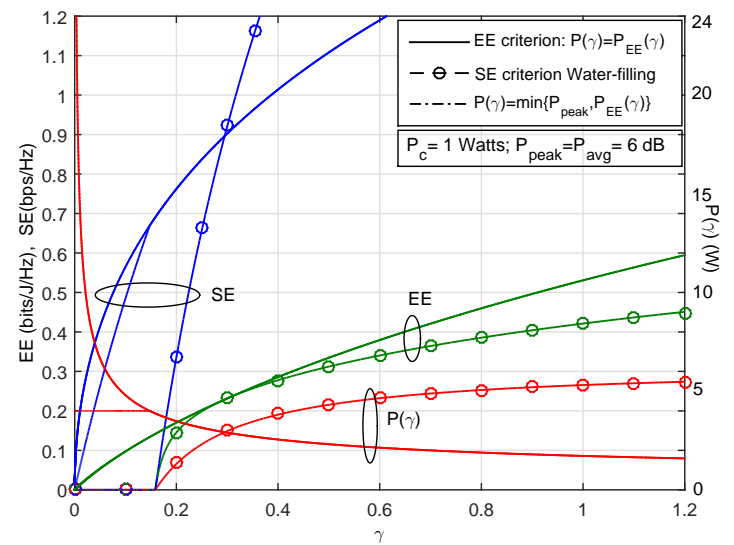

Figure 3: Instantaneous EE and SE vs $\gamma$.

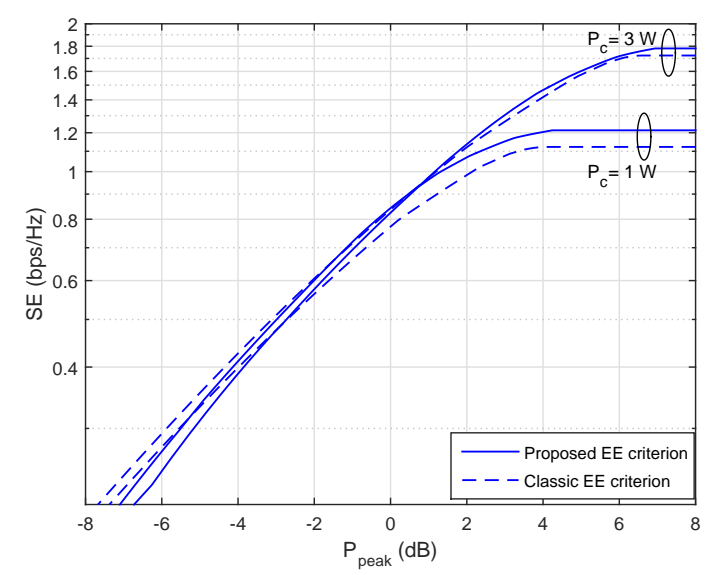

Figure 4: Comparison of the corresponding average SE of the proposed EE and the classical EE.

Consequently, the corresponding EE and SE are only affected in this region of $\gamma$ when a peak power is set. The maximum loss in SE is about $44 \%$ when $\gamma=0.04$. We also highlight, in Fig. 3, the difference in performances between adopting the EE criterion and adopting the SE criterion, with average power constraint $P_{\text {avg }}=6 \mathrm{~dB}$. In this case, we compare the $\mathrm{EE}$ criterion in continuous curves with the SE criterion, in dashed curves. Note that the chosen value of $P_{\text {avg }}=6 \mathrm{~dB}$ exceeds $\mathbb{E}_{\gamma}\left[P_{E E}(\gamma)\right]$. Hence, the corresponding EE matches the EE with no power limitation. As can be seen in Fig. 3, the $E E_{S E_{-} \text {criterion }}$ reaches the $E E_{E E_{-} \text {criterion }}$ for a reduced interval of $\gamma$ but is always below it. In terms of SE, at low values of $\gamma$, the $S E_{E E_{-} \text {criterion }}$ is higher than $S E_{S E_{-} \text {criterion, }}$, but as $\gamma$ increases, the $S E_{S E_{-} \text {criterion }}$ becomes higher. In Fig. 4, we perform a comparison between the proposed average $\mathrm{EE}$ and the classical EE in terms of achieved SE with peak power constraint. As shown in Fig. 4, the proposed average EE provides a higher SE than the one corresponding to the classical EE, in the high power regime $\left(P_{\text {peak }}>-3 \mathrm{~dB}\right.$ for $P_{c}=1 \mathrm{~W}$ and $P_{\text {peak }}>1 \mathrm{~dB}$ for $P_{c}=3 \mathrm{~W}$ ). In Fig. 5, the performances with peak power constraint are presented for different values of $P_{c}$. We first show that the EE increases

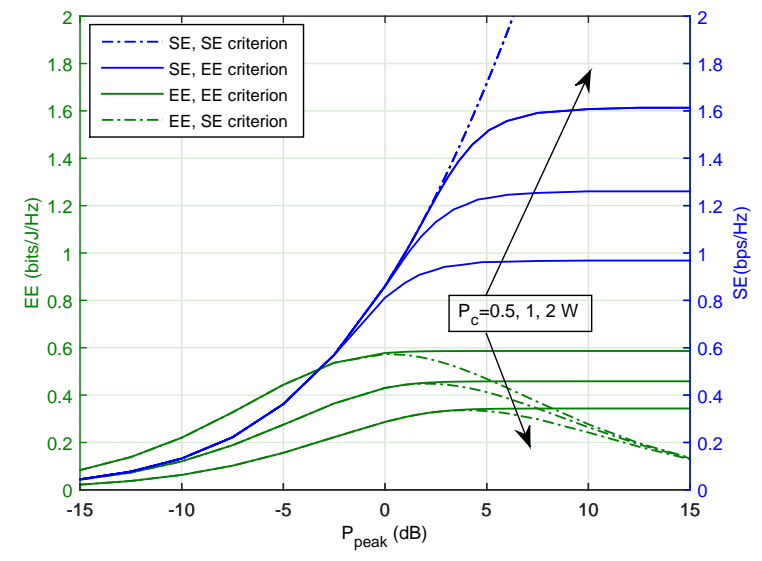

Figure 5: Average EE and SE vs $P_{\text {peak }}$ with perfect CSI.

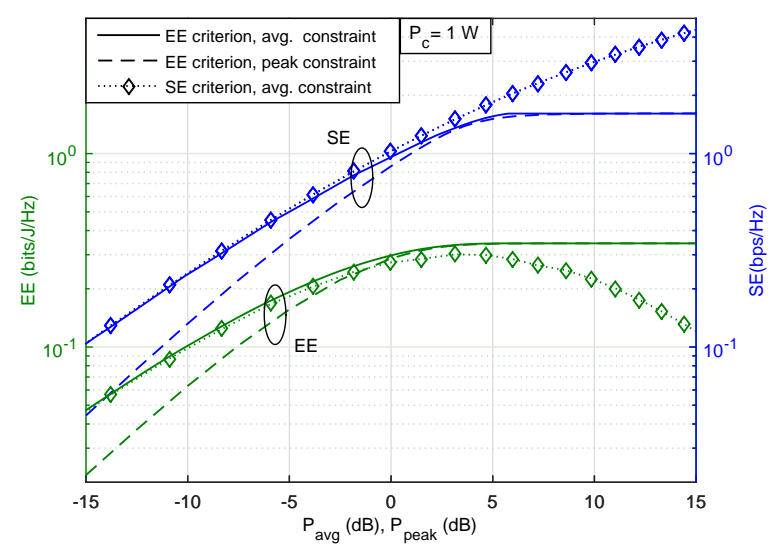

Figure 6: Average EE and SE vs $P_{\text {avg }}$ with perfect CSI.

and reaches a certain asymptote as shown in (13) and for a fixed $P_{c}$, there is no better energy efficient transmission that can be achieved beyond this asymptote. In fact, when the EE criterion is considered, there is no need to increase the power once the EE is close to its asymptote. Also, as expected form (III-B3), when $P_{c}$ increases, the EE decreases and the SE increases. In addition, we note that at low values of $P_{\text {peak }}$, the performances of the EE criterion and the SE criterion are identical since the active power is $P_{\text {peak }}$. However, the EE at low $P_{\text {peak }}$ varies with $P_{c}$ whereas the $\mathrm{SE}$ is constant which reflects a higher sensitivity of the EE with respect to $P_{c}$ at low power. In Fig. 6, we present the EE and SE when an average power constraint is adopted. The SE criterion, in this case, is based on the water-filling power allocation. Note that the values of $\mathbb{E}_{\gamma}\left[P_{E E}(\gamma)\right]$ is $4.24 \mathrm{~dB}$. We show that, for $P_{\text {avg }} \leq 4.24 \mathrm{~dB}$, the $E E_{E E_{-} \text {criterion }}$ is slightly above the $E E_{S E_{-} \text {criterion }}$ but we have the inverse for the SE. As $P_{\text {avg }}$, exceeds $4.24 \mathrm{~dB}$, the $E E_{S E_{-} \text {criterion }}$ decreases towards zero whereas $E E_{E E_{-} \text {criterion }}$ remains at its highest value given by (13). From $S E$ perspective, the $E E_{S E_{-} \text {criterion }}$ stagnates compared to the $S E_{S E_{-} \text {criterion }}$ which is the price to pay when considering an $\mathrm{EE}$ criterion. Hence, when the $\mathrm{EE}$ is the criteria to consider, there is no need for the power to 


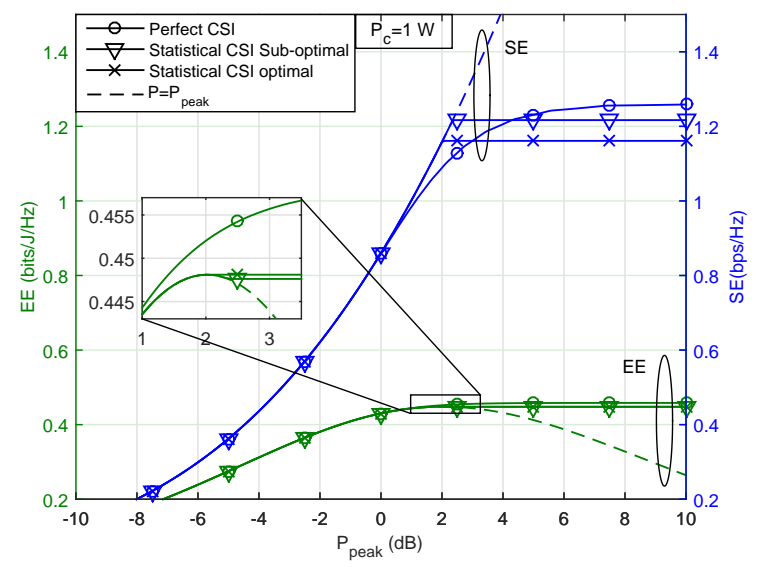

Figure 7: Average EE and average SE vs $P_{\text {peak }}$ with statistical CSI.

exceed $4.24 \mathrm{~dB}$, i.e., $\mathbb{E}_{\gamma}\left[P_{E E}(\gamma)\right]$ since it will result in a waste of power with no enhancement in both $\mathrm{EE}$ and SE performances. We also present the performances when a peak power constraint is adopted, and as can be seen in Fig. 6, the average constrained power performs better at low values.

In Fig. 7, we analyze the performances of the optimal and sub-optimal power allocation schemes described in (46) and (48) as function of $P_{\text {peak }}$, respectively, when the CSI is not available. Note that since the average constraint becomes a peak constraint when the CSI is not available as mentioned in Section V.A.3 and V.B.2, the following performance of the peak power constraint applies to the average constraint. The Fig.7 shows that the sub-optimal and power allocation schemes give a very close EE. Hence, it is worthwhile adopting the suboptimal power since it is easy to compute and does not require finding a root of the integral equation (46). In addition, we show that the gap between perfect CSI and statistical CSI EE is very limited, in fact the loss is about $0.01 \mathrm{bits} / \mathrm{J} / \mathrm{Hz}$ which present $3 \%$ of the perfect CSI EE. In terms of SE, we notice that the statistical CSI SE is better than the perfect CSI SE between 1 and $4 \mathrm{~dB}$. This striking result is not surprising since the focus is on EE criteria. Also, at high $P_{\text {peak }}$, the optimal and sub-optimal statistical CSI SE present a loss of $3 \%$ and $8 \%$, respectively, compared to the perfect CSI SE. Consequently, the CSI unavailability does not deteriorate the performances and simple power schemes based on the channel mean can achieve acceptable performances. In addition, the CSI is needed in the high power regime to achieve better performance which is different from the SE maximization problem where the CSI is required at low power regime. In Fig. 8, we present the CR performance when both peak power and interference constraints are adopted. We compare between 3 cases in which either the PU is absent $\left(I_{\text {peak }} \rightarrow \infty\right)$ or present $\left(I_{\text {peak }}=0\right.$ $\mathrm{dB}$ and $3 \mathrm{~dB}$ ). We notice that $I_{\text {peak }}$ slightly affects the EE in the high power regime (i.e., $P_{\text {peak }} \geq 0 \mathrm{~dB}$ ). For instance, for $P_{\text {peak }}=6 \mathrm{~dB}$, the EE gain between $I_{\text {peak }}=0 \mathrm{~dB}$ and $I_{\text {peak }} \rightarrow \infty$ is about $12 \%$ where it is $5 \%$ for $I_{\text {peak }}=3 \mathrm{~dB}$. From another side, the gap between the perfect CSI and the

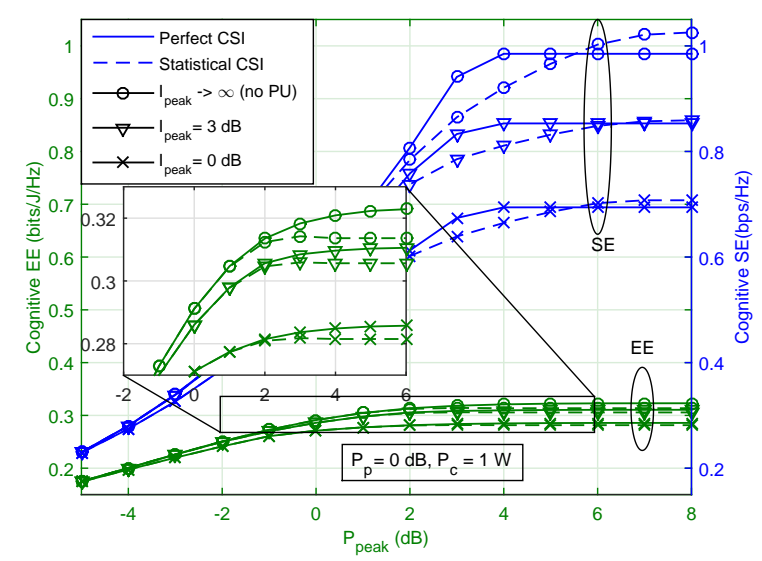

Figure 8: Cognitive EE and SE with peak power and interference constraints.

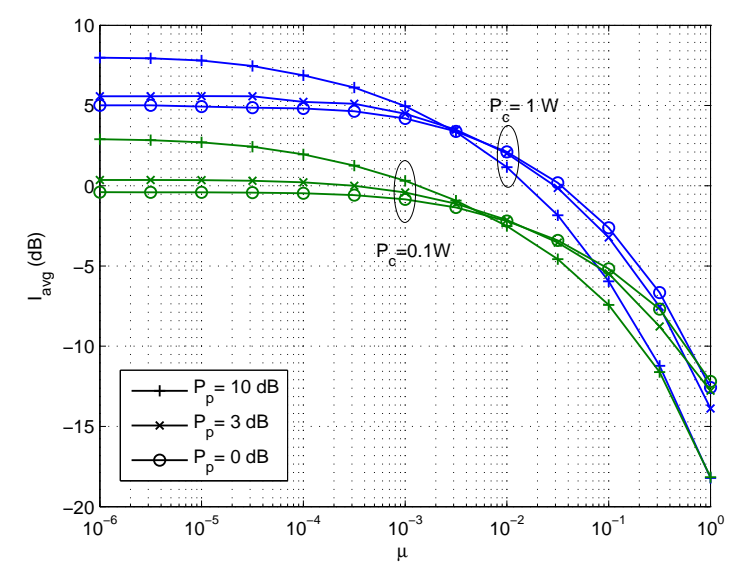

Figure 9: $I_{\text {avg }}$ as a function of $\mu$.

statistical CSI EE performance is about $1.5 \%$ which means that the absence of CSI has a limited effect on the EE when the power is only adapted to the channels' statistics. In Fig. 9, we present the relation between the average interference threshold $I_{\text {avg }}$, and the Lagrange multiplier associated to the interference constraint, $\mu$ presented in the equation (40). In fact, in order to find the solution of (40), for a given $I_{a v g}$, we, first, search for the corresponding $\mu$, then find the solution, this figure presents a lookup table that facilitates finding the optimal power for average interference constrained problems. We show in this figure that the maximum value of $I_{\text {avg }}$ that prevents outage transmission depends on the primary power $P_{p}$ and the circuit power $P_{c}$. For example if $P_{p}=0 \mathrm{~dB}$ and $P_{c}=0.1 W$, there will be no transmission if $I_{a v g}>0 \mathrm{~dB}$.

In Fig. 10, the cognitive rates when average power and interference constraints as a function of $P_{\text {avg }}$ with different values of $I_{\text {avg }}$. We notice that similarly to the peak constraints in Fig.8, the differences between the EE when there is no PU and when $I_{\text {avg }}=-2.5 \mathrm{~dB}$ is 0.05 bits $/ \mathrm{J} / \mathrm{Hz}$ (reduction of $10 \%$ ) and when $I_{a v g}=-5 \mathrm{~dB}$ it is equal to $0.1 \mathrm{bits} / \mathrm{J} / \mathrm{Hz}$ (reduction of 21\%). Whereas on the SE the reductions form 


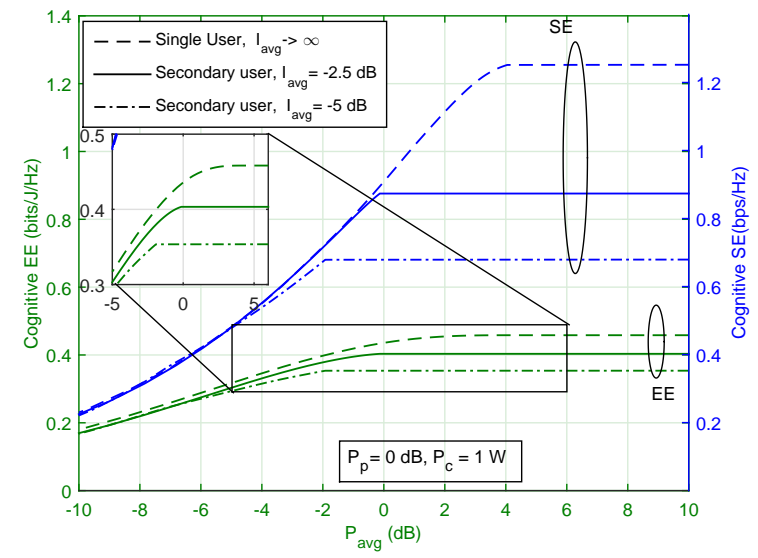

Figure 10: Cognitive EE and SE with average power and interference constraints.

the no PU performance are higher; $30 \%$ for $I_{a v g}=-2.5 \mathrm{~dB}$ and $45 \%$ for $I_{a v g}=-5 \mathrm{~dB}$.

\section{CONCLUSION}

In this paper, we analyzed the energy efficiency of powerconstrained systems and present explicit expressions of the optimal power. We first studied a point to point system and found the explicit power that maximizes the EE despite its non-convexity. Then, we present optimal and sub-optimal power expressions when only statistical CSI-T is available. We extend our work to spectrum sharing cognitive radio (CR) systems where either a peak or an average power constraint is adopted. In the numerical results, we showed that the performances depend mainly on the circuit power and that the CSI-T absence slightly affects the EE compared to the perfect CSI-T EE. Unlike SE-maximized design where absence of CSI-T is detrimental in the low power regime, EE-maximized design suffers a performance loss in the high power regime in the absence of CSI-T. In the CR setting, we showed that the effect of the interference constraint is minimal on the $\mathrm{EE}$ compared to the SE.

\section{Appendix A : Proof of (3)}

We first note the $E E$ is a continuous and differentiable function for all the values of $P \geq 0$. Also since it was mentioned in Section III.A that it has a global maxima, it will necessarily correspond to a root of the first-order derivative. This derivative is computed as follows

$$
\frac{\partial E E}{\partial P}=\frac{\gamma}{\log (2)(\gamma P+1)\left(P+P_{c}\right)}-\frac{\log (\gamma P+1)}{\log (2)\left(P+P_{c}\right)^{2}}
$$

The eventual optimal power is given when $\frac{\partial E E}{\partial P}=0$ which means

$$
\begin{aligned}
& (\gamma P+1) \log (\gamma P+1)-(\gamma P+1)=\gamma P_{c}-1 \\
& \Leftrightarrow X(\log (X)-1)=\gamma P_{c}-1(\text { where } X=\gamma P+1) \\
& \Leftrightarrow e^{\log (X)}(\log (X)-1)=\gamma P_{c}-1 \\
& \Leftrightarrow e^{\log (X)-1}(\log (X)-1)=\frac{\gamma P_{c}-1}{e} \\
& \Leftrightarrow \log (X)-1=W\left(\frac{\gamma P_{c}-1}{e}\right) \\
& \Leftrightarrow X=e^{W\left(\frac{\gamma P_{c}-1}{e}\right)+1} \\
& \Leftrightarrow P_{E E}=\frac{1}{\gamma}\left(e^{1+W\left(\frac{\gamma P_{c}-1}{e}\right)}-1\right)
\end{aligned}
$$

Since, we found only one root of first-order derivative, this root corresponds necessarily to the maxima of EE. From (59), we have

$$
E E=\frac{\gamma}{\log (2)(\gamma P+1)}=\frac{\gamma}{\log (2) e^{1+W\left(\frac{\gamma P_{c}-1}{e}\right)}}
$$

and the corresponding rate is $S E=\log _{2}(X)=\frac{W\left(\frac{\gamma P_{c}-1}{e}\right)+1}{\log (2)}$.

\section{Appendix B : Obtaining Equation (5)}

We have $E E^{*}=\frac{S E^{*}}{P_{c}+P_{E E}}$, which gives

$$
E E^{*} P_{c}=S E^{*}-E E^{*} P_{E E} .
$$

From (4), we have $E E^{*}=\frac{\gamma}{\log (2) 2^{S E^{*}}}$. Thus, $E E^{*} P_{E E}=$

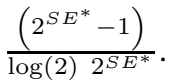

Equation (68) gives $E E^{*} P_{c}=S E^{*}-\frac{\left(2^{S E^{*}}-1\right)}{\log (2) 2^{S E^{*}}}$, which implies that $E E^{*}=\frac{1}{P_{c}}\left(S E^{*}-\frac{1-2^{-S E^{*}}}{\log (2)}\right)$.

\section{Appendix C : Concavity of EE When $P(\gamma) \leq P_{E E}(\gamma)$}

We first recall that $P_{E E}$ is the unique maximum of EE since it is the unique root of its derivative, $E E^{\prime}$ as was shown in Appendix A. Consequently, the sign of $E E^{\prime}$ between 0 and $P_{E E}$ is the same and is given by any point, for example for $P=0$ we have $E E^{\prime}(0)=\frac{\gamma}{P_{c}}>0$. Then, for $P(\gamma) \leq P_{E E}(\gamma)$ we have $E E^{\prime}(P)>0$. Now we compute the second derivative of EE with respect to $P$ and we can show that it can be expressed as a function of the first derivative as follows:

$$
E E^{\prime \prime}(P)=-\frac{1}{P_{c}+P}\left(\frac{\gamma^{2}}{(1+\gamma P)^{2} \log (2)}+2 E E^{\prime}(P)\right) \text {. }
$$

Since $E E^{\prime}(P) \geq 0$ for $P(\gamma) \leq P_{E E}(\gamma)$ then from (69), we have $E E^{\prime \prime}(P)<0$ which means that $E E$ is a concave function for $P(\gamma) \leq P_{E E}(\gamma)$.

\section{REFERENCES}

[1] L. Sboui, Z. Rezki, and M.-S. Alouini, "On energy efficient power allocation for Power-Constrained systems," in Proc. of the 25th IEEE International Symposium on Personal Indoor and Mobile Radio Communications (PIMRC'14), Washington, DC, USA, Sep. 2014, pp. 1954 1958.

[2] "Spectrum policy task force," Federal Communications Commission, Tech. Rep. ET Docket no. 02-135, Nov. 2002. 
[3] J. Mitola and G. Q. Maguire, "Cognitive radio: Making software radios more personal," IEEE Personal Communications Magazine, vol. 6, no. 4, pp. 13-18, Aug. 1999.

[4] S. Haykin, "Cognitive radio: Brain-empowered wireless communications," IEEE Journal on Selected Areas in Communications, vol. 23, no. 2, pp. 201-220, Feb. 2005.

[5] A. Fehske, G. Fettweis, J. Malmodin, and G. Biczok, "The global footprint of mobile communications: The ecological and economic perspective," IEEE Communications Magazine, vol. 49, no. 8, pp. 55-62, Aug. 2011.

[6] Gartner, "Gartner estimates ICT industry accounts for 2 percent of global CO2 emissions," http://www.gartner.com/newsroom/id/503867, 2007.

[7] G. Rittenhouse, S. Goyal, D. T. Neilson, and S. Samuel, "Sustainable Telecommunications," in Technical Symposium at ITU Telecom World (ITU WT), 2011, pp. 19-23.

[8] Z. Hasan, H. Boostanimehr, and V. Bhargava, "Green cellular networks: A survey, some research issues and challenges," IEEE Communications Surveys Tutorials, vol. 13, no. 4, pp. 524-540, Fourth 2011.

[9] ERICSSON, "Sustainable energy use in mobile communications," White Paper, 2007.

[10] K. Pentikousis, "In search of energy-efficient mobile networking," IEEE Communications Magazine, vol. 48, no. 1, pp. 95-103, 2010.

[11] I. F. Akyildiz, W. Su, Y. Sankarasubramaniam, and E. Cayirci, "Wireless sensor networks: A survey," Computer networks, vol. 38, no. 4, pp. 393 422, 2002.

[12] A. Goldsmith, S. Jafar, I. Maric, and S. Srinivasa, "Breaking spectrum gridlock with cognitive radios: An information theoretic perspective," Proceedings of the IEEE, vol. 97, no. 5, pp. 894-914, May 2009.

[13] R. Zhang, "On peak versus average interference power constraints for protecting primary users in cognitive radio networks," Wireless Communications, IEEE Transactions on, vol. 8, no. 4, pp. 2112-2120, Apr. 2009.

[14] D. Feng, C. Jiang, G. Lim, L. J. Cimini Jr, G. Feng, and G. Y. Li, "A survey of energy-efficient wireless communications," IEEE Communications Surveys \& Tutorials, vol. 15, no. 1, pp. 167-178, 2013.

[15] A. Goldsmith and P. Varaiya, "Capacity of fading channels with channel side information," IEEE Transactions on Information Theory, vol. 43, no. 6, pp. 1986-1992, Nov. 1997.

[16] C. Shannon, "Communication in the presence of noise," Proceedings of the IRE, vol. 37, no. 1, pp. 10-21, Jan. 1949.

[17] Y. Chen, S. Zhang, S. Xu, and G. Y. Li, "Fundamental trade-offs on green wireless networks," IEEE Communications Magazine, vol. 49, no. 6 , pp. 30-37, 2011.

[18] S. Verdu, "Spectral efficiency in the wideband regime," IEEE Transactions on Information Theory, vol. 48, no. 6, pp. 1319-1343, June 2002.

[19] G. Li, Z. Xu, C. Xiong, C. Yang, S. Zhang, Y. Chen, and S. Xu, "Energyefficient wireless communications: Tutorial, survey, and open issues," IEEE Wireless Communications Magazine, vol. 18, no. 6, pp. 28-35, Dec. 2011

[20] X. Chen, R. Q. Hu, G. Wu, and Q. C. Li, "Tradeoff between energy efficiency and spectral efficiency in a delay constrained wireless system," Wireless Communications and Mobile Computing, pp. 1530-8677, 2014.

[21] C. Xiong, G. Y. Li, S. Zhang, Y. Chen, and S. Xu, "Energy-and spectralefficiency tradeoff in downlink OFDMA networks," IEEE Transactions on Wireless Communications, vol. 10, no. 11, pp. 3874-3886, 2011.

[22] O. Onireti, F. Héliot, and M. A. Imran, "Closed-form approximation for the trade-off between energy efficiency and spectral efficiency in the uplink of cellular network," in European Wireless ConferenceSustainable Wireless Tech. (EW'11), Vienna, Austria, 2011, pp. 1-6.

[23] H. Kwon and T. G. Birdsall, "Channel capacity in bits per joule," IEEE Journal of Oceanic Engineering, vol. 11, no. 1, pp. 97-99, 1986.

[24] F. Meshkati, H. Poor, S. Schwartz, and N. B. Mandayam, "An energyefficient approach to power control and receiver design in wireless data networks," IEEE Transactions on Communications, vol. 53, no. 11, pp. 1885-1894, Nov 2005.

[25] S. Cui, A. J. Goldsmith, and A. Bahai, "Energy-constrained modulation optimization," IEEE Transactions on Wireless Communications, vol. 4, no. 5, pp. 2349-2360, 2005.

[26] C. Isheden, Z. Chong, E. Jorswieck, and G. Fettweis, "Framework for link-level energy efficiency optimization with informed transmitter," IEEE Transactions on Wireless Communications, vol. 11, no. 8, pp. 2946-2957, 2012.

[27] D. Persson, T. Eriksson, and E. Larsson, "Amplifier-aware multipleinput multiple-output power allocation," IEEE Communications Letters, vol. 17 , no. 6, pp. 1112-1115, June 2013.
[28] S. Mikami, T. Takeuchi, H. Kawaguchi, C. Ohta, and M. Yoshimoto, "An efficiency degradation model of power amplifier and the impact against transmission power control for wireless sensor networks," in IEEE Radio and Wireless Symposium, Jan 2007, pp. 447-450.

[29] C. Isheden and G. P. Fettweis, "Energy-efficient multi-carrier link adaptation with sum rate-dependent circuit power," in IEEE Global Telecommunications Conference (GLOBECOM'10), Miami, Florida, USA, 2010, pp. 1-6.

[30] M. Naeem, K. Illanko, A. Karmokar, A. Anpalagan, and M. Jaseemuddin, "Optimal power allocation for green cognitive radio: fractional programming approach," IET Communications,, vol. 7, no. 12, 2013.

[31] R. M. Corless, G. H. Gonnet, D. E. Hare, D. J. Jeffrey, and D. E. Knuth, "On the Lambert W function," Advances in Computational Mathematics, vol. 5, no. 1, pp. 329-359, 1996.

[32] L. Venturino, C. Risi, S. Buzzi, and A. Zappone, "Energy-efficient coordinated user scheduling and power control in downlink multi-cell OFDMA networks," in Proc. of the IEEE International Symposium on Personal Indoor and Mobile Radio Communications (PIMRC'13), London, UK, Sept 2013, pp. 1655-1659.

[33] R. M. Corless, D. J. Jeffrey, and D. E. Knuth, "A sequence of series for the Lambert W function," in Proc. of the International Symposium on Symbolic and Algebraic Computation (ISSAC'97), Maui, Hawaii, USA, 1997, pp. 197-204.

[34] S. Boyd and L. Vandenberghe, Convex Optimization. Cambridge University Press, 2004

[35] X. Kang, Y.-C. Liang, A. Nallanathan, H. Garg, and R. Zhang, "Optimal power allocation for fading channels in cognitive radio networks: Ergodic capacity and outage capacity," IEEE Transactions on Wireless Communications, vol. 8, no. 2, pp. 940-950, Feb. 2009.

[36] C.-X. Wang, X. Hong, H.-H. Chen, and J. Thompson, "On capacity of cognitive radio networks with average interference power constraints," IEEE Transactions on Wireless Communications, vol. 8, n.o. 4, pp. 1620-1625, April 2009

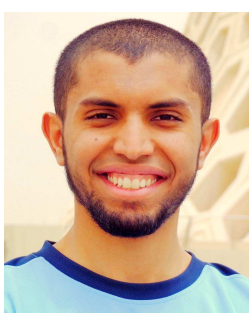

Lokman Sboui (S'11) was born in Cairo, Egypt. He received the Diplome d'Ingénieur degree with honors from Ecole Polytechnique de Tunisie (EPT), La Marsa, Tunisia, in 2011, the MS degree from King Abdullah University of Science and Technology (KAUST) in May 2013. He is currently a Ph.D. candidate in the Electrical Engineering program of KAUST. His current research interests include: performance of cognitive radio systems, low SNR communication, MIMO communication, Relaying performances, energy efficient power allocation and green wireless sensor networks

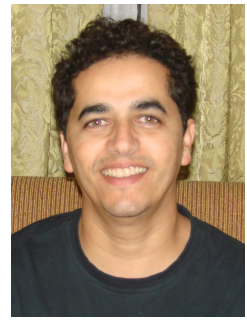

Zouheir Rezki (S'01, M'08) was born in Casablanca, Morocco. He received the Diplome d'Ingénieur degree from the École Nationale de l'Industrie Minérale (ENIM), Rabat, Morocco, in 1994, the M.Eng. degree from École de Technologie Supérieure, Montreal, Québec, Canada, in 2003, and the Ph.D. degree from École Polytechnique, Montreal, Québec, in 2008, all in electrical engineering. From October 2008 to September 2009, he was a postdoctoral research fellow with Data Communications Group, Department of Electrical and Computer Engineering, University of British Columbia. He is now a research scientist at King Abdullah University of Science and Technology (KAUST), Thuwal, Makkah Province, Saudi Arabia. His research interests include: performance limits of communication systems, cognitive and sensor networks, physicallayer security, and low-complexity detection algorithms.

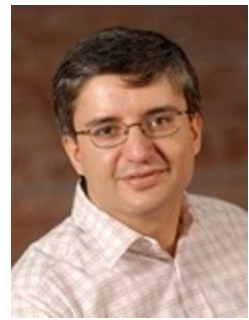

Mohamed-Slim Alouini (S'94, M'98, SM'03, F'09) was born in Tunis, Tunisia. He received the Ph.D. degree in Electrical Engineering from the California Institute of Technology (Caltech), Pasadena, CA, USA, in 1998. He served as a faculty member in the University of Minnesota, Minneapolis, MN, USA, then in the Texas A\&M University at Qatar, Education City, Doha, Qatar before joining King Abdullah University of Science and Technology (KAUST), Thuwal, Makkah Province, Saudi Arabia as a Professor of Electrical Engineering in 2009. His current research interests include the modeling, design, and performance analysis of wireless communication systems. 